\title{
Code/Code comparison of impurity transport in EMC3 and DIVIMP ${ }^{\text {« }}$
}

\author{
K. Schmid ${ }^{\mathrm{a}, *}$, T. Lunt ${ }^{\mathrm{a}}$, W. Zhang ${ }^{\mathrm{a}}$ \\ ${ }^{a}$ Max-Planck-Institut für Plasmaphysik, Boltzmannstraße 2, D-85748 Garching b. München Germany
}

\begin{abstract}
Recently the global impurity migration code WallDYN was coupled to the 3D edge transport code EMC3-Eirene in order to make WallDYN applicable to 3D, non-toroidally symmetric, geometries. As part of the coupling a new impurity transport module was implemented which, in contrast to the existing impurity module, takes the inertia of the impurity into account, making it more suitable for heavy impurities like W. This new "kinetic" impurity transport module uses the same physics as the 2D trace impurity transport code DIVIMP and therefore, to check the correctness of the implementation, a code/code benchmark was performed comparing the transport of Be- and W-impurities in EMC3 and DIVIMP. The comparison was performed for a toroidally symmetric plasma that could be handled both by EMC3 and DIVIMP. Both codes were setup such that they would treat the transport of the impurities as similar as possible. Then the re-distribution of Be and $\mathrm{W}$ launched from different positions around the poloidal circumference of the first wall was calculated in both codes. To compare the resulting material re-distribution data $\left(\equiv R_{i, j}\right)$ and judge the significance of any differences, $R_{i, j}$ was used in WallDYN calculations which model the evolution of the first wall due to erosion, deposition, re-erosion and re-deposition. These WallDYN calculations, performed using the $R_{i, j}$ from EMC3 or DIVIMP, showed the same major erosion and deposition areas and it can therefore be concluded that the new kinetic impurity transport module is implemented correctly and now allows to make DIVIMP like transport calculations using EMC3 in 3D.
\end{abstract}

\section{Introduction}

During the interaction of the scrape off layer (SOL) plasma with the first wall components, material is eroded by sputter or chemical erosion and enters the plasma as an impurity species. The migration of these impurities in the plasma and their re-deposition to form layers are a challenge for long term plasma operation: Firstly, during the growth of these layers large amounts of hydrogen species $(\mathrm{H})$ can be retained in these layers via co-deposition [1]. Secondly due to growth under energetic particle bombardment, these layers are under large mechanical stresses,

\footnotetext{
Poster PB029

* K. Schmid

Email address: klaus.schmid@ipp.mpg.de (K. Schmid)
}

making them prone to delamination. If such a layer fractures and enters the plasma the subsequent radiative energy loss can potentially disrupt the discharge. Therefore, making predictions on material erosion, migration in the plasma and the location of re-deposition, where layers may form, is a key information for planning the operation of future fusion devices like ITER.

In previous studies the global impurity migration code WallDYN [2, 3] has been used to interpret Be layer formation in the JET-ILW campaign and make predictions for ITER.

WallDYN is a wall dynamics code that computes the time evolution of the first wall composition, the resulting evolution of the first wall erosion and self-consistently computes 
the resulting changes in the impurity influxes onto the wall. It maintains a global material balance and allows to determine the changes in the impurity influxes into the plasma due to layer deposition. To do that WallDYN requires information from edge transport codes on how eroded material is re-distributed by the plasma: If material is launched from location $\mathrm{i}$ on the wall, what is the first location $\mathrm{j}$ on the wall it impinges on and at which charge state $\mathrm{Z}$ does the impact occur. This is the redistribution matrix $R_{i, j}$ for charge state Z. In addition, the edge transport codes have to provide the wall plasma parameters (fluxes and temperatures) to derive the poloidal, and for 3D cases toroidal, distribution of material erosion. For further details the reader is referred to $[2,3]$. Thus WallDYN can be thought of as a post processor for trace impurity transport calculations that handles the complex chain of multiple impurity transport steps: erosion, deposition/reflection, re-erosion and re-deposition

Up to now WallDYN used the 2D trace impurity transport code DIVIMP [4] to model the migration of impurities in the SOL and was thus limited to quasi 2D toroidally symmetric cases. To improve the predictions by including shadowed areas (e.g. poloidal limiters) a 3D model of the scrape off layer and 3D impurity transport is needed. Therefore WallDYN has been coupled [5] to the 3D edge transport code EMC3 [6]-Eirene [7] (三 WallDYN3D). As part of this effort a new trace impurity transport module was implemented [5] in EMC3 that mimics the transport model in DIVIMP. As was shown in [5] this new model should be better suited to simulate the transport of heavy impurities like W, since it includes the influence of the particle's inertia on transport. In order to test the new transport model, trace impurity transport simulations were performed for the same $2 \mathrm{D}$, toroidally symmetric ITER plasma scenario using DIVIMP \& EMC3-Eirene. Since the 2D background plasma solution used in DIVIMP is based on SOLPS4.3 [8] and the 3D solution was calculated by EMC3-Eirene [9], the background parameters do not match perfectly in the $2 \mathrm{D}$ and $3 \mathrm{D}$ cases. In particular the background flow pattern was different in several locations. The temperature and density matched quite well in the upstream regions but at the target region, probably due to the lack of detachment physics in EMC3, the plasma temperatures are much higher in EMC3 than in the 2D SOLPS based solution. Still the resulting material redistribution data ( $\equiv R_{i, j}$ ) shows the same major features. An even better match is achieved if the the 2D plasma is mapped onto the $3 \mathrm{D}$ grid resulting in virtually identical background plasmas in $2 \mathrm{D}$ and $3 \mathrm{D}$. For the $R_{i, j}$ based on this "mapped" background plasma, WallDYN calculations were performed. Comparing these calculations, differing only in the $R_{i, j}$ data taken either from DIVIMP or EMC3, showed that in both cases the erosion and deposition patterns matched. It can therefore be concluded that the new kinetic impurity transport module is implemented correctly and now allows to make DIVIMP like transport calculations in EMC3 in 3D.

\section{Comparing background plasma solutions}

In order to compare the two codes two plasma solutions, one in $2 \mathrm{D}$ and one in $3 \mathrm{D}$, are needed. To select a plasma background that is available both for EMC3 and DIVIMP the database of ITER plasma solutions was searched. Thereby the details of the plasma solution were of no concern, they just had to be as identical as possible. The SOLPS H-mode case i-dib-0903-1514-00g has been used for trace impurity studies in DIVIMP before [10]. For these calculations the computational grid was extended to reach all the way to wall, by applying the "onion skin method" (OSM) approach in the gap between the SOLPS grid and the wall, as outlined in [8]. The same case was modelled in 3D using EMC3-Eirene [9] with a grid that is based on the same magnetic equilibrium and is using the same toroidally symmetric first wall contour. Fig 1 shows an overlay of both grids in the divertor region. The first wall contour is identical to the outer edge 
of the red DIVIMP grid. The EMC3 grid feature three zones (blue SOL, green CORE, black PFZ) and extends far beyond the first wall contour, these outer regions are passed to EIRENE for neutral particle modelling while solving the plasma solution. In toroidal direction the simulation domain spans $20^{\circ}$ and the $3 \mathrm{D}$ grid has 17 steps in toroidal direction. At the resulting toroidal boundary cells, periodic boundary conditions are applied to model the entire $360^{\circ}$ circumference. Comparing the two grids, the separatrix and X-point match perfectly but the dome region is slightly different: In the DIVIMP grid the pumping ducts below the divertor dome were resolved, whereas in the EMC3 grid these features are missing. Therefore in the impurity transport calculations these differing regions in the grid and wall definition have to be excluded from the comparison. However we consider this as a minor limitation and therefore chose case i-dib-0903-1514-00g for the code/code comparison.

It should be pointed out that no plasma background solutions were generated as part of this work but we relied on the plasma solutions and computational grids provided to us by the original authors of [8] and [9].

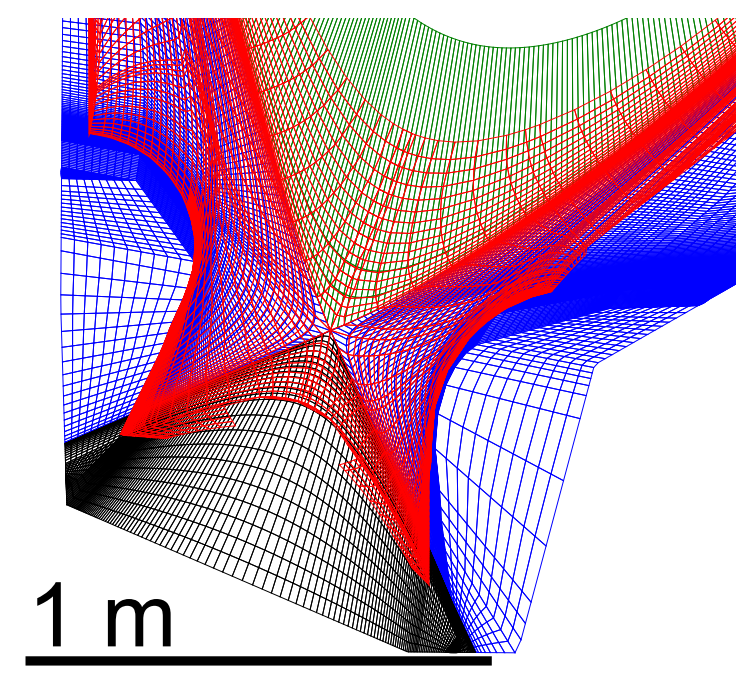

Figure 1: Overlay of the 2D DIVIMP (red) with a single poloidal plane of the 3D grid used in EMC3. The X-point and separatrix location match but the divertor dome is slightly different in the $2 \mathrm{D}$ grid.
To compare the plasma solution on the two grids, radial rays are cast through the grids and the plasma parameters (electron density $n_{e}$, temperature $T_{e}$ and flow mach number M) along the intersection with the grid cells are recorded and are then compared vs. the distance along the rays. In addition, the plasma parameters at the wall contour are compared which, later in the WallDYN calculation, determine the impurity sources via erosion. In Fig 2 the location of the test rays is shown. As an example of

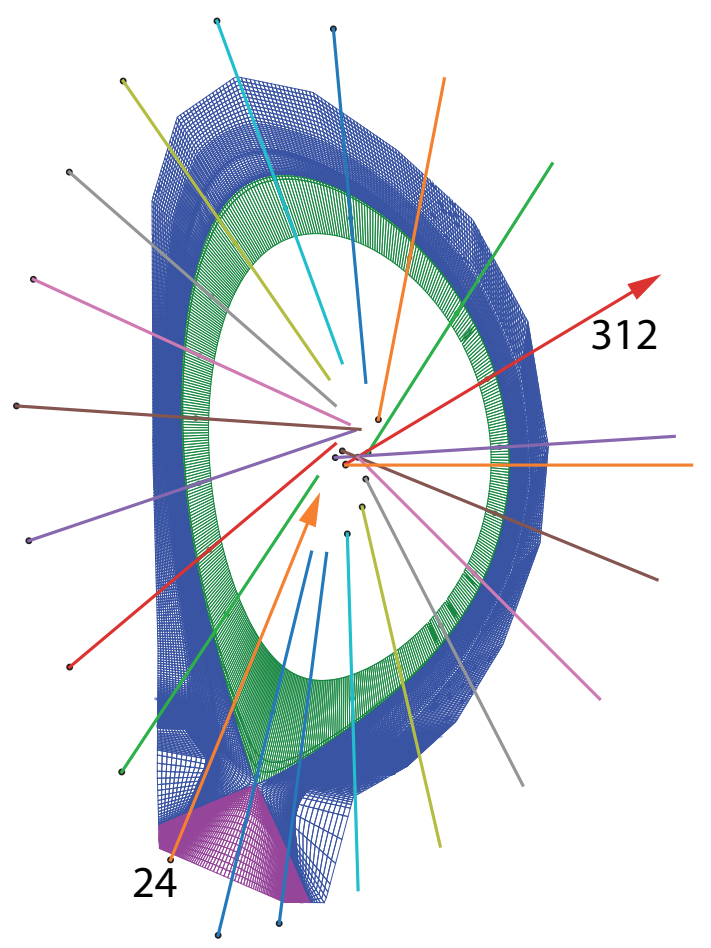

Figure 2: Location of the test rays used to probe the plasma parameters in the two solutions. In DIVIMP rays are cast radially through the single poloidal plane whereas in EMC3 at each poloidal plane (i.e. at each toroidal location) rays are cast radially and the values of $n_{e}, T_{e}$ and $\mathrm{M}$ in the intersected cells are recorded

the plasma comparison Fig. 3 and 4 show the $n_{e}, T_{e}$ and mach flow values along the rays labeled 24 and 312 in Fig 2 . The match between $n_{e}$ and $T_{e}$ is generally rather good. In contrast the comparison of the background plasma flow pattern shown in Fig 4 is generally not so good, while the shape is generally similar the absolute values differ quite significantly. The comparisons of the two plasma solutions performed along the other rays in Fig. 2 show a similar 
level of consistency. Differences in the flow pattern are
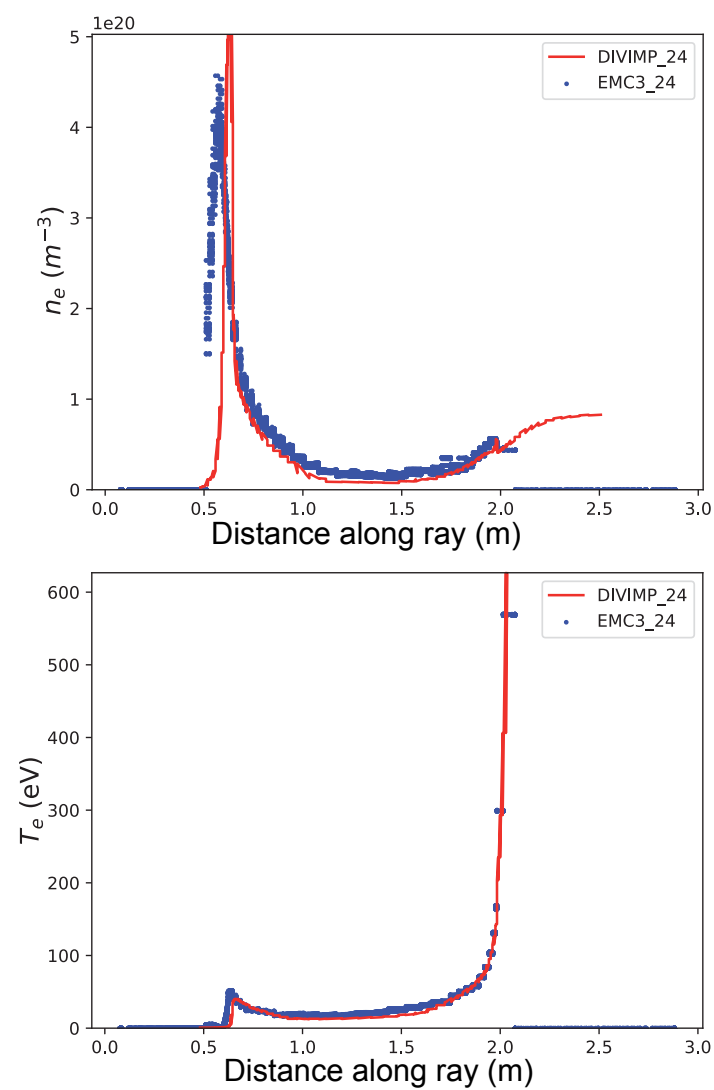

Figure 3: Example of the $n_{e}, T_{e}$ values along the ray labled 24 in Fig. 2 in the EMC3 [9] and the DIVIMP plasma solution [8]. The horzontal-axis is position of the grid cell centre along the length of the intersecting ray

a problem for the code/code comparison of impurity migration, since the mach flow determines the friction force between the impurities and the main plasma ions, which is one of the dominating forces determining the impurity transport (see [11] chapter 6.5). To compare the plasma parameters at the wall obtained from the 2D SOLPS/OSM plasma solution used in DIVIMP, with the values from the 3D plasma solution from EMC3-Eirene, the first wall was subdivided into 51 wall elements as shown in Fig. 5. The black solid line in Fig. 5 is the wall contour used in EMC3, so it does not feature the pump ducts below the divertor dome present in the DIVIMP wall contour. The differences can be seen in the insert in Fig. 5 which shows a zoom into the divertor region. There the DIVIMP contour
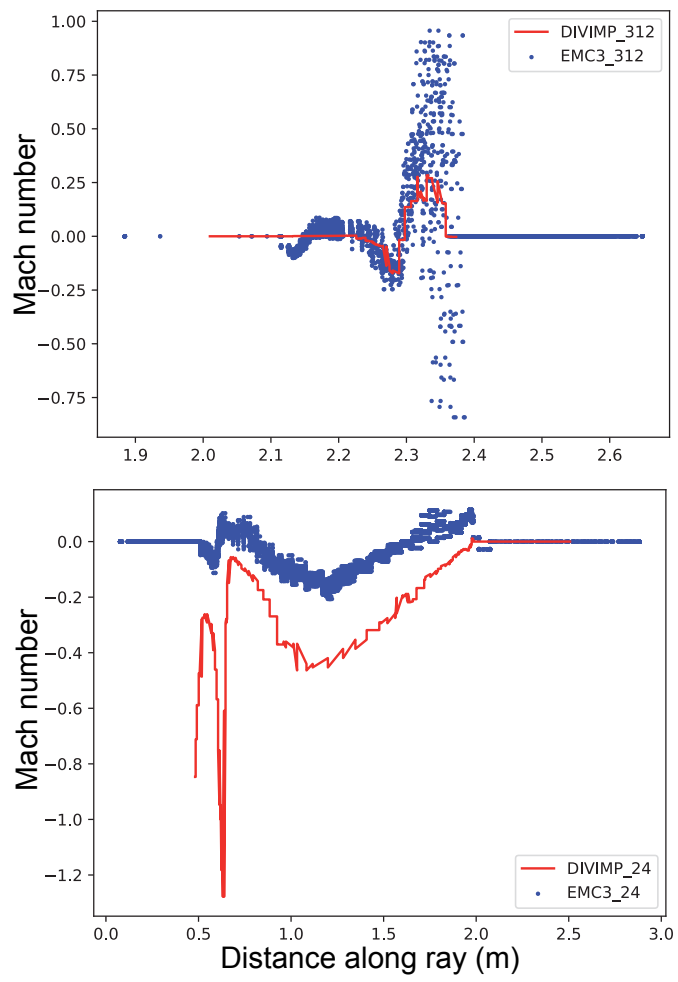

Figure 4: Example of the mach flow values along the rays labled 24 and 312 in Fig. 2 in the EMC3 [9] and the DIVIMP plasma solution [8]. The horzontal-axis is position of the grid cell centre along the length of the intersecting ray 
is depicted in red dashed lines. Therefore the plasma parameters and also the impurity migration results for wall indices 29 to 31 and 36 to 39 cannot be directly compared due to the different wall geometry.

For DIVIMP the wall plasma in the poloidal plane can be obtained directly, whereas for EMC3-Eirene the values are first averaged along the toroidal direction. Since the plasma is toroidally symmetric this toroidal averaging merely reduces the monte-carlo noise (EMC3 uses a monte-carlo method to solve the fluid equations [6]) and does not lead to loss of generality.

Comparing the wall plasma solution in Fig. 6 shows

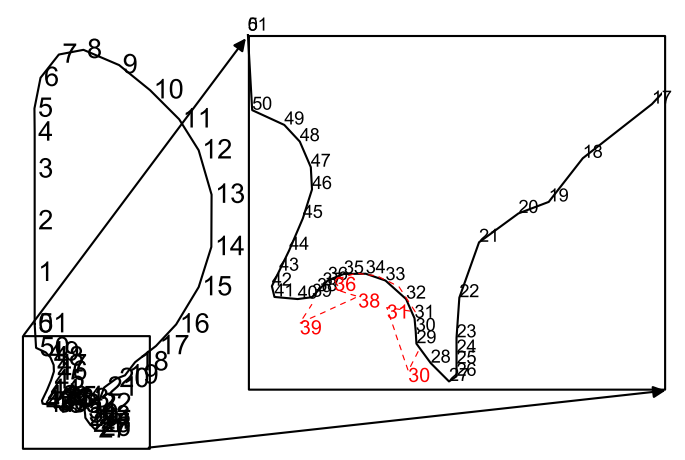

Figure 5: The subdivision of the first wall in the poloidal plane. For EMC3 the additional toroidal coordinate is projected into one poloidal plane. Also shown is the DIVIMP dome wall contour with the additional pump duct feature. The wall index numbers given are 0 based

large differences in the divertor region (wall indices 20 to 50). The reason being that EMC3 lacks the ability to model detached plasmas, since it does not include recombination processes of the majority plasma ions. Therefore the EMC3 solution in the divertor is an attached solution, resulting in much higher plasma temperatures. There are also differences in the electron temperature at the low field side of the main wall (wall indices 10 to 15 ). This is due to the fact that the SOLPS solution does not reach all the way to wall at these locations and the plasma there was derived using the "onion skin method" (OSM) approach [8] assuming a weak decay of $T_{e}$ from the edge of the SOLPS solution grid radially outward to the wall. In EMC3-Eirene this gap to the wall does not exist and therefore no assumptions on the radial fall off enter into the calculation and the resulting $T_{e}$ value at the wall is part of the global background plasma solution. In summary the plasma so-

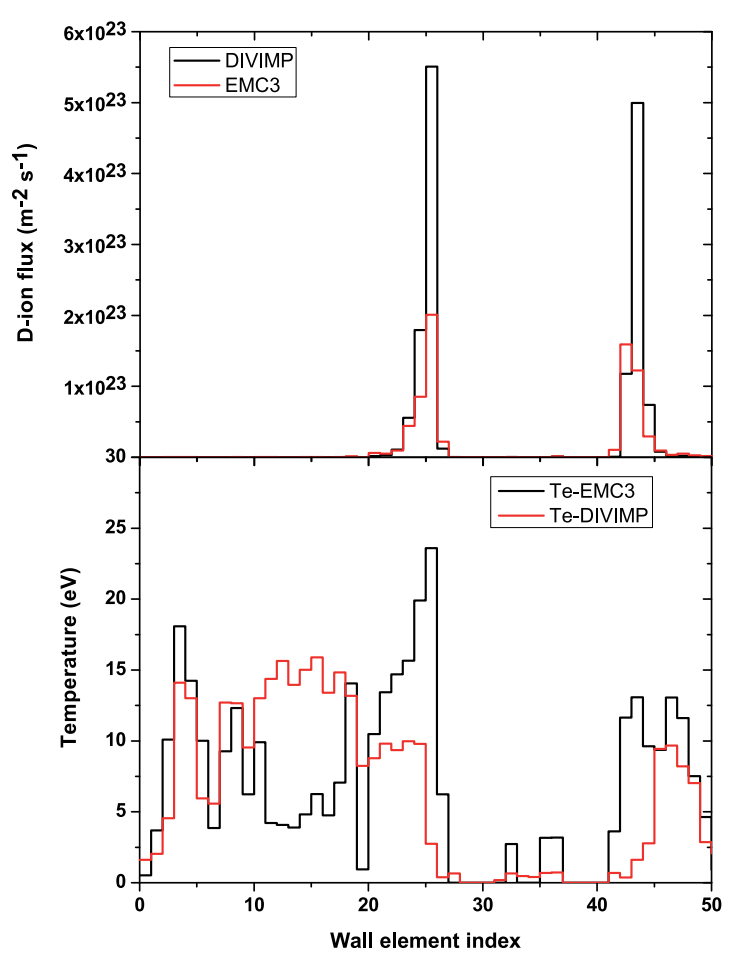

Figure 6: Comparison of the main plasma parameters at the wall as obtained from the SOLPS/OSM plasma solution used in DIVIMP and the EMC3-Eirene result

lutions are rather similar taking into account the different approaches used in the codes, in particular the simplified plasma physics applied in EMC3-Eirene compared to SOLPS. However for a code/code comparison on impurity migration a better match in the background plasma is desirable, in particular a better match of the flow pattern is needed. To that end the SOLPS/OSM plasma solution was mapped onto each poloidal plane in the 3D grid used in EMC3. This mapping was performed by linearly interpolating the plasma parameters positioned at the cell centres ( $R, Z$ coordinates) of the $2 \mathrm{D}$ solution, at the location of the $3 \mathrm{D}$ cell centers $(R, Z, \Phi)$ at each toroidal angle $\Phi$ in the $3 \mathrm{D}$ grid. The resulting match of the plasma param- 
eters was checked in the same manner as above by casting radial rays and the match was perfect in all location. The impurity migration calculations described in section 3 were then performed on both the "Native" plasma solutions (as computed by SOLPS/OSM and EMC3-Eirene respectively) and on the so called "Mapped" plasma solutions (derived by mapping the $2 \mathrm{D}$ plasma onto the $3 \mathrm{D}$ grid).

\section{Impurity migration calculations}

Based on the selected/generated plasma solutions, impurity transport calculations were performed using both DIVIMP and EMC3. These calculations were performed in trace limit i.e. there was no feedback of the resulting impurity density on the plasma solution. From these calculations the required $R_{i, j}$ data for $\mathrm{W}$ and Be impurities was derived, which is needed to later perform a full WallDYN calculation for the ITER first wall. The two codes were setup such that they should apply the same physics model for impurity transport and not to best mimic the conditions in an ITER discharge. This is necessary since not all features available in DIVIMP are also available in EMC3. To that end prompt re-deposition was turned off in DIVIMP and the impurities were set to be reflected at the SOL/Core interface, thus confining transport to the main SOL only. Of course the same ADAS data for ionisation and recombination rate coefficients was used in both codes.

As required to determine $R_{i, j}$, the impurities were launched from each of the 51 wall elements shown in Fig. 5 in a separate calculation. For EMC3 the launch occurred homogeneously along the toroidal direction thus assuming toroidal symmetry as in DIVIMP. The particles were all launched with the same angular distribution in both codes and a fixed initial energy of $2 \mathrm{eV}$ for Be and $4 \mathrm{eV}$ for $\mathrm{W}$ as expected from the Thomson Distribution of the energy for physically sputtered particles with surface binding energies of Be: $3.2 \mathrm{eV}$ and $\mathrm{W}: 8.86 \mathrm{eV}$. The particles are launched as neutrals in both codes. In EMC3 the neutrals follow a straight line until they're ionised or impact on another wall element. In addition DIVIMP also includes momentum changing collisions for the neutrals with the background ions. In these collisions the direction of the velocity in deflected randomly by 90 degrees in $3 \mathrm{D}$ space. In Fig. 7 the resulting neutral densities in front of the source location are shown for the example of a launch of $\mathrm{W}$ from the top of the machine (wall index 10). To be able to compare the values on the different grids, Fig. 7 shows a contour plot of the neutral density values located at the R,Z cell centres, for DIVIMP from the single poloidal plane and for EMC3 from all poloidal planes at the different $\Phi$ values in toroidal direction. The match in the neutral density contours is good, with the highest densities close to the upper left edge of the wall launch location. This match is important, since this neutral density distribution is also the source distribution of the ions that are then followed in the rest of the migration calculation.

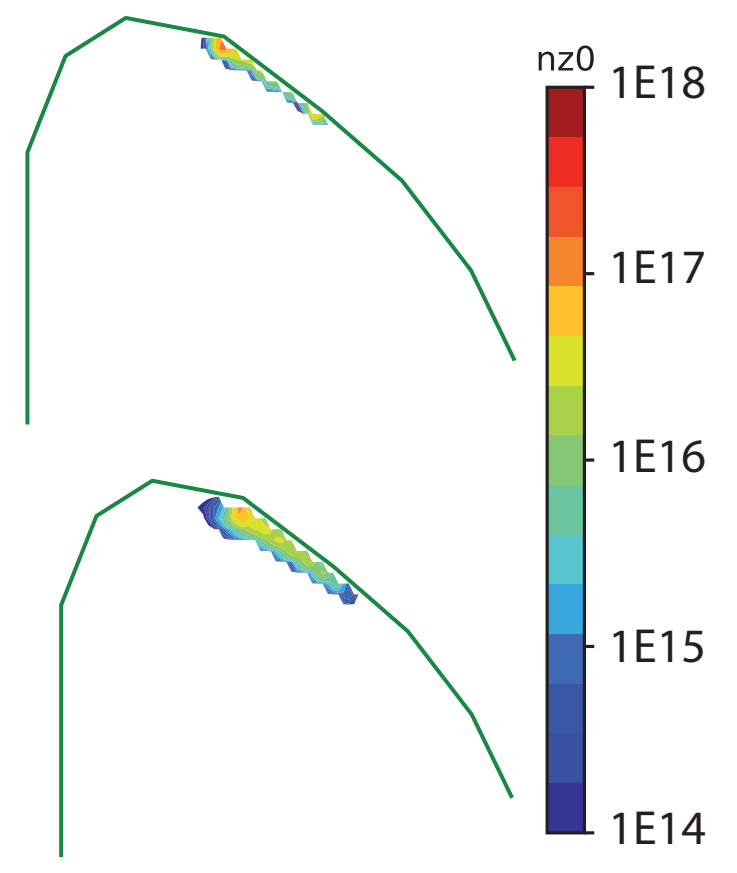

Figure 7: Example of the comparison of the neutral density in front of the launch location at wall index 10 in Fig. 5

After ionisation the particles are transported in parallel 
along the field lines due to forces arising mainly from friction with the background plasma and by temperature gradients (see also [11] chapter 6.5). This parallel transport is implemented identically in DIVIMP and in the newly implemented kinetic transport module in EMC3 [5]. The impurities also migrate perpendicularly to the field lines by diffusive steps with diffusion coefficient of $1\left(\mathrm{~m}^{2} \mathrm{~s}^{-1}\right)$. In DIVIMP these diffusion steps only occur in radial direction whereas in EMC3, real 2D diffusion perpendicular to the local magnetic field direction is computed. A further difference between the codes is the sequence of events: In DIVIMP a single time step for the ion transport consists of first performing the perpendicular step, then the parallel step and finally the particle charge state is updated based on the respective rate coefficients for ionisation and recombination at the grid location AFTER the parallel position update is completed. In EMC3, in each time step, also the particle first undergoes perpendicular transport, but the parallel step is divided into sub-steps if the particle transitions cell boundaries: The particle charge state is updated based on the LOCAL values of the rate coefficients in the current cell.

The parallel and perpendicular transport of the impurities including changes in charge state due to further ionisation or recombination steps is followed until the impurity is lost to the wall. In DIVIMP the particle is deposited at the wall location nearest to the last grid cell. In contrast in EMC3 the particle is terminated if it enters a cell that intersects a wall component and this cell deposition is recorded. The final deposition location on the first wall components is then determined by a post processing step, where the cell deposition information is projected along the field line onto the wall component surfaces. This can shift the final deposition location both poloidally and toroidally due to the field line pitch angle. In both codes the wall deposition is recorded in a charge state resolved manner. From this deposition information the charge state resolved redistribution matrix $R_{i z, i, j}$ which denotes the fraction of impurities launched from wall element $i$ that end up on wall element $j$ at charge state $i z$. This matrix can then be compared to obtain information about the differences in the transport calculations in these two codes.

In Fig. 8 the charge state integrated matrix $\sum_{i z} R_{i z, i, j}$ is shown for Be as computed by DIVIMP and EMC3 using either the EMC3-Eirene calculated background or the SOLPS/OSM solution mapped onto the 3D grid.

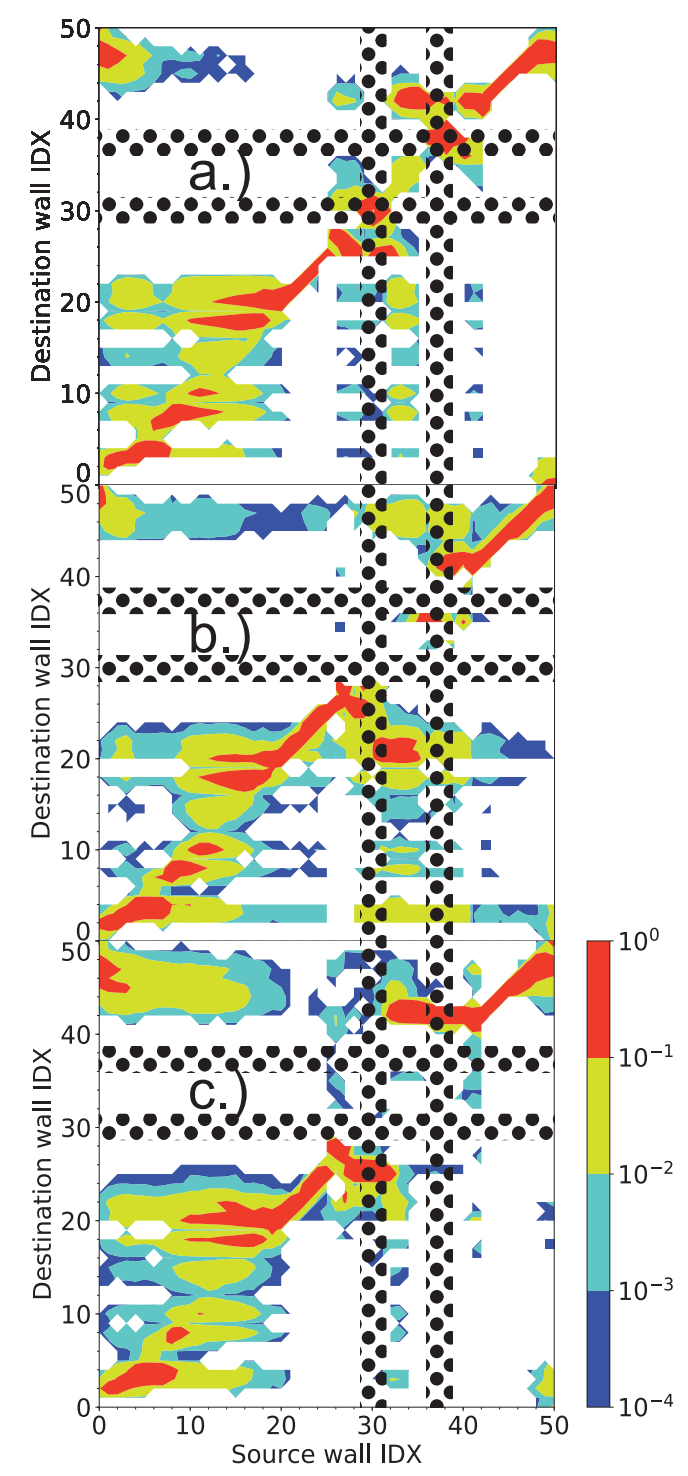

Figure 8: Computed charge state integrated redistribution matrices by a.) DIVMP b.) EMC3 with the EMC3-Eirene calculate background and c.) EMC3 with SOLPS/OSM solution mapped onto the 3D grid. The dotted areas mark the regions affected by the different wall contour below the divertor dome

In all three types of calculations the main qualitative 
features are the same: A strong diagonal corresponding to local re-deposition close to the point of origin. Also the divertor entrance baffles (outer baffle at wall index 20 to 25 and the inner baffle 45 to 50) are main locations of re-deposition. Looking at a more detailed level shows differences in the re-distribution of Be launched from the divertor dome baffle for case b.) and c.) in Fig. 8. For the EMC3-Eirene based plasma solution b.) Be emanating from the dome (wall index $\approx 34$ ) predominately ends up on the top of the outer baffle (wall index $\approx 20$ ) whereas for the SOLPS/OSM solution in a.) and its mapping onto the $3 \mathrm{D}$ grid in c.) Be ends up below the inner strike point (wall index $\approx 41$ ). Generally case b.) results in more redeposition of Be launched from within the divertor volume (wall indices 20 to 50) on the top of the baffles. This can be attributed to the different temperatures and flow patterns in the divertor in case b.) compared to case a.) and c.). Generally, as expected, the mapped SOLPS/OSM solution in case c.) best matches the DIVIMP solution in case a.). None the less there are still differences between cases a.) and c.) despite the essentially identical background plasma solution. Looking at the charge state resolved $R_{i z, i, j}$ shows that the discrepancies in $\sum_{i z} R_{i z, i, j}$ are mainly due to differences in the higher charge states, an issue that becomes even more apparent for $\mathrm{W}$ which quickly charges up to ionisation states of $\approx 8+$ and higher.

The comparison for $\mathrm{W}$ impurity transport is shown in Fig. 9 and 10 where the differences in the redistribution matrix between DIVIMP and EMC3 with the mapped SOLPS/OSM solution are compared for low and high charge states. While the match in $R_{+1, i, j}$ for $\mathrm{W}^{+1}$ is quite good, it becomes worse for higher charge states e.g. $\mathrm{W}^{+9}$. For EMC3, high charge states are present at the inner (wall index 40 to 50) and outer (wall index 20 to 24) divertor baffle, whereas for DIVIMP this contribution is not present. The difference in charge state evolution is likely due to the different sequence of transport and charge state change events during each time step as described above.

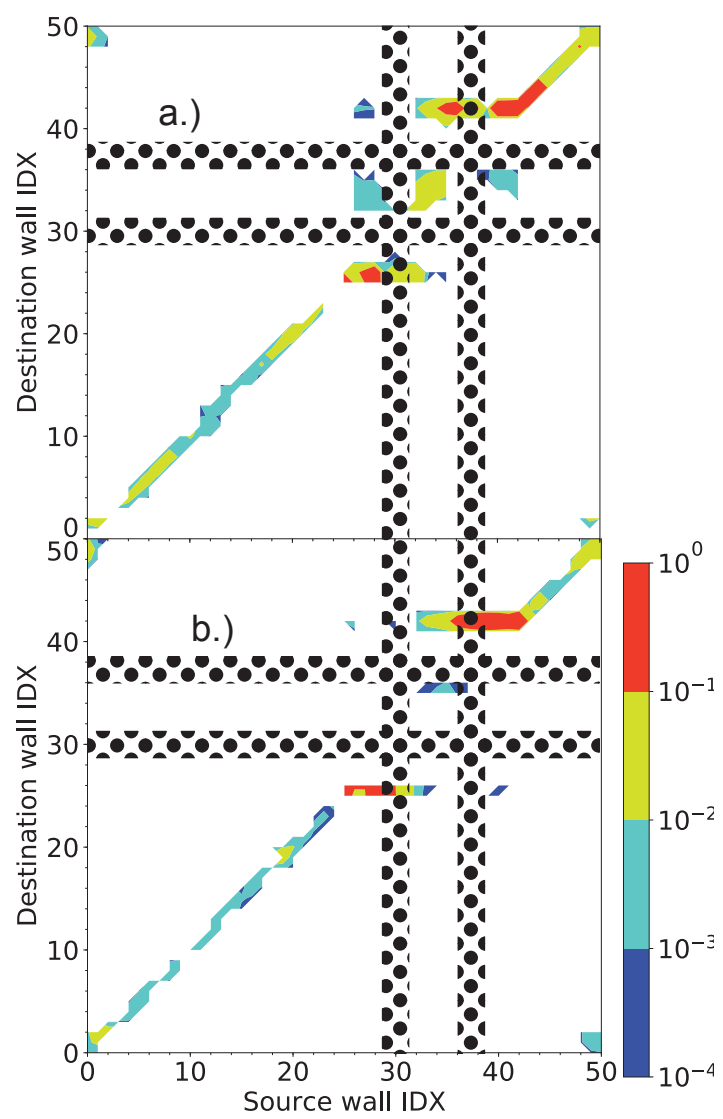

Figure 9: Computed charge state resolved redistribution matrix for $\mathrm{W}^{+1}$ by a.) DIVMP b.) EMC3 with the SOLPS/OSM solution mapped onto the $3 \mathrm{D}$ grid. The dotted areas mark the regions affected by the different wall contour below the divertor dome 


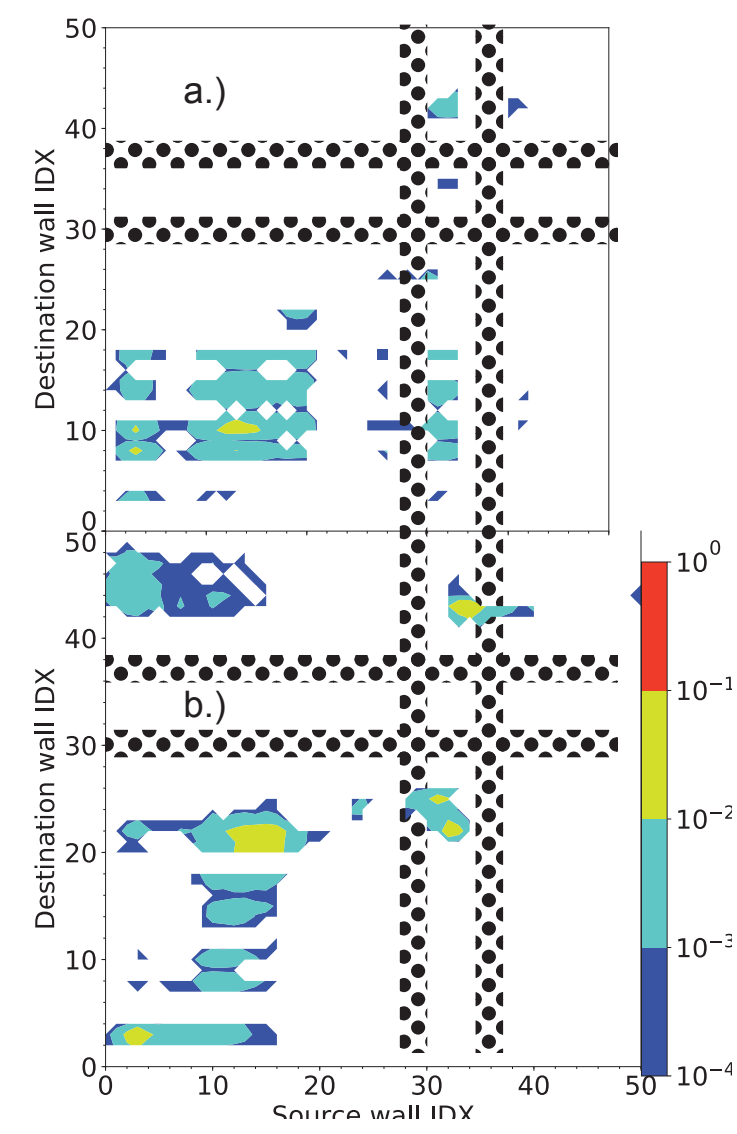

Figure 10: Computed charge state resolved redistribution matrix for $\mathrm{W}^{+9}$ a.) by DIVMP b.) EMC3 with the SOLPS/OSM solution mapped onto the $3 \mathrm{D}$ grid. The dotted areas mark the regions affected by the different wall contour below the divertor dome

\section{WallDYN calculations}

While a comparison of the $\sum_{i z} R_{i z, i, j}$ showed rather similar results in both DIVIMP and EMC3 based trace impurity transport calculations, in particular for the mapped SOLPS/OSM background plasma solution, judging whether these differences are significant or not requires an appropriate measure: The goal of the code/code comparison is to assure that the new kinetic impurity transport module in EMC3 can be used to derive reliable $R_{i z, i, j}$ matrices for WallDYN that are comparable to what DIVIMP would yield. Therefore WallDYN calculations are performed, differing only in the $R_{i z, i, j}$ matrices taken either from DIVIMP or from EMC3 with the mapped SOLPS/OSM background plasma solution.

WallDYN is a global wall composition dynamics code, that works essentially as post processor for $R_{i z, i, j}$ matrices taken from any given trace impurity transport code and wall plasma conditions which are taken from the background plasma solution $R_{i z, i, j}$ was calculated from. It couples erosion, migration, re-deposition and re-erosion processes to model the time evolution of the first wall composition thereby maintaining a global material balance. A detailed description of its concept and applications can be found in $[2,3,10]$. The WallDYN calculation used the same settings as in [10] and the wall evolution was followed for 500 seconds until all erosion deposition rates on the wall tiles were constant i.e. an equilibrium was reached.

In Fig. 11 the output of the WallDYN calculation is compared. The graphs labeled DIVIMP and EMC3 only differ by the redistribution matrix used, all the other input values, in particular the wall plasma parameters, were the same in both calculations. In Fig. 11 a.) the equilibrium wall concentration is shown and both codes yield the same areas where close Be layers form, with the exception of the top of the outer baffle (wall index $\approx 21$ ): There DIVIMP predicts a closed Be layer whereas EMC3 only yields partial surface coverage. The net Be deposition rate, negative meaning net erosion and positive net depo- 


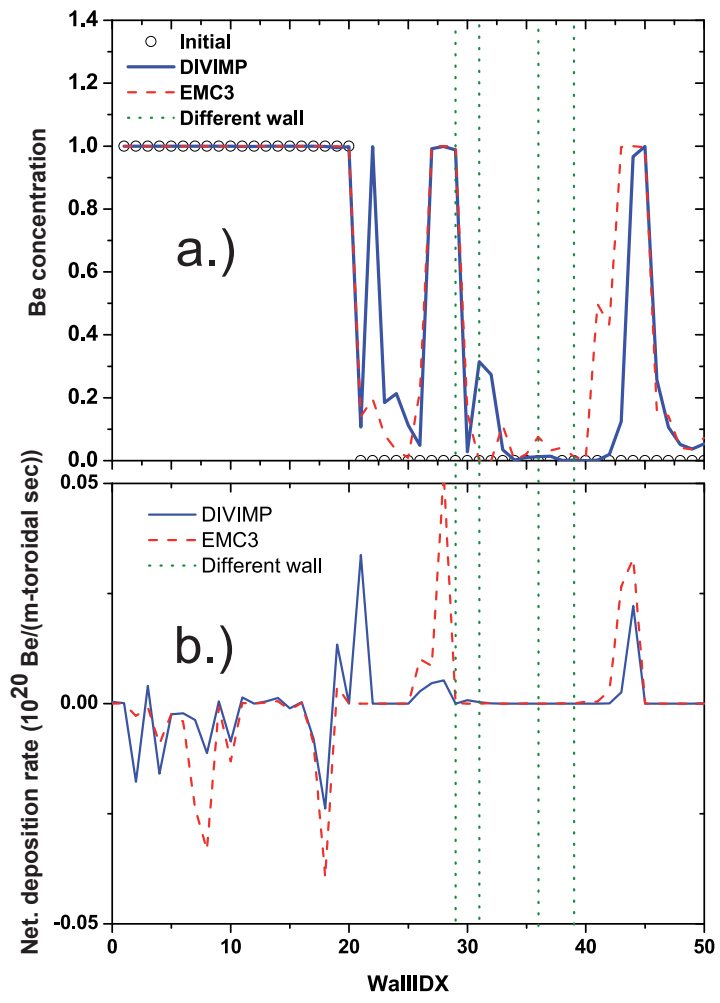

Figure 11: Equilibrium first wall composition a.) and net Be deposition rate b.) as calculated by WallDYN after $500 \mathrm{sec}$. of continuous plasma operation. sition of Be, is shown in Fig. 11 b.). The units Be/(mtoroidal $\times \mathrm{sec})$ result from multiplying the computed Be net deposition/erosion rates with units of $\mathrm{Be} /\left(\mathrm{m}^{2} \mathrm{~s}\right)$ with the poloidal length $\mathrm{m}$ of the wall elements. For both codes the basic erosion/deposition patterns are similar but the absolute magnitude differs significantly. With $R_{i z, i, j}$ taken from DIVIMP net erosion occurs at multiple location on the main chamber Be wall, whereas for EMC3 erosion predominantly occurs at the top of the machine and close to the outer divertor entrance. Net deposition occurs below the strike points for both codes and DIVIMP predicts additional deposition on top of the outer divertor baffle. Based on the deposition rates where Be layers grow, WallDYN also derives fuel retention rates via co-deposition, by multiplying the Be layer growth rate, with the expected $\mathrm{D} / \mathrm{Be}$ ratio in these layers taken from scaling laws [1]. The D/Be ratio thereby is a function temperature, particle energy and Be deposition rate. Comparing the total D retention rates computed based on $R_{i z, i, j}$ from the two codes one finds a factor 2 difference with DIVIMP suggesting more D co-depostion.

In summary despite the underlaying differences in the two codes they yield rather similar impurity migration patterns. In particular the new kinetic impurity transport module that was implemented in EMC3 [5] works correctly. Thus EMC3 can now be used for 3D impurity migration studies.

\section{Conclusions}

A code/code benchmark comparing the new "kinetic" impurity transport module in EMC3 to the DIVIMP trace impurity transport code was performed for a toroidally symmetric background plasma. The 2D background plasma for DIVIMP was taken from SOLPS and the 3D plasma solution was calculated by EMC3 trying to match the SOLPS result. The two background plasma solutions only partially matched. In particular the flow pattern was different in some locations and the divertor plasma was 
very different in the EMC3 solution due to lack of detachment. Still the resulting migration patterns show the same major features. Using a mapping of the $2 \mathrm{D}$ solution onto the $3 \mathrm{D}$ grid, yielded a much better match in the migration patterns. To judge the significance of the remaining differences, the re-distribution data $R_{i, j}$ was used in WallDYN calculations. The erosion and deposition patterns calculated based on $R_{i, j}$ taken from DIVIMP or EMC3 respectively were very similar allowing to conclude that the new "kinetic" impurity transport module in EMC3 works correctly. Thus EM3 can now be used for interpreting 3D impurity migration experiments also for heavy impurities like W for which the new "kinetic" transport model is well suited. In the future one could also feedback the evolution of the impurity influx into the plasma, as calculated by WallDYN, into the plasma solving iteration in EMC3. Thus including the evolution of the first wall in the equilibrium solution of the SOL plasma.

\section{Acknowledgment}

This work has been carried out within the framework of the EUROfusion Consortium and has received funding from the Euratom research and training programme 20142018 and 2019-2020 under grant agreement No 633053. The views and opinions expressed herein do not necessarily reflect those of the European Commission.
[1] G. D. Temmerman, M. Baldwin, R. Doerner, D. Nishijima, K. Schmid, Nucl. Fusion 48 (2008) 075008.

[2] K. Schmid, M. Reinelt, K. Krieger, J. Nucl. Mater. 415 (2011) 284.

[3] K. Schmid, K. Krieger, S. W. Lisgo, G. Meisl, S. Brezinsek, J. E. Contributors, J. Nucl. Mater. 463 (2015) 66.

[4] P. Stangeby, J. D. Elder, J. Nucl. Mater. 196-198 (1992) 258.

[5] K. Schmid, T. Lunt, 3d global impurity transport modeling with walldyn and emc3-eirene, Nuclear Materials and Energy 17 (2018) 200.

[6] Y. Feng, F. Sardei, J. Kisslinger, P. Grigull, K. McCormick, D. Reiter, Contrib. Plasma Phys. 1-3 (2004) 57.

[7] D. Reiter, M. Baelmans, P. Börner, The eirene and b2-eirene codes, Fusion Science and Technology 47 (2) (2005) 172. doi:10.13182/FST47-172.

[8] S. Lisgo, A. Kukushkin, R. Pitts, D. Reiter, J. Nucl. Mater. 438 (2013) 580.

[9] W. Zhang, R. Bilato, T. Lunt, A. Messiaen, R. Pitts, S. Lisgo, X. Bonnin, V. Bobkov, D. Coster, Y. Feng, P. Jacquet, J. Noterdaeme, Scrape-off layer density tailoring with local gas puffing to maximize icrf power coupling in iter, Nuclear Materials and Energy 19 (2019) 364.

[10] K. Schmid, K. Krieger, S. Lisgo, G. Meisl, S. Brezinsek, J. Contributors, Nuclear Fusion 55 (2015) 053015.

[11] P. C. Stangeby, The plasma boundary of magnetic fusion devices, IOP Pulishing, 2000. 